\title{
COMONOTONIC APPROXIMATIONS FOR THE SUM OF LOG UNIFIED SKEW NORMAL RANDOM VARIABLES: APPLICATION IN FINANCE AND ACTUARIAL SCIENCE
}

\author{
Arjun K. Gupta*a and Mohammad A. Azizb \\ aDepartment of Mathematics and Statistics, Bowling Green State University, U.S.A. \\ bDepartment of Mathematics, University of Wisconsin-Eau Claire, U.S.A.
}

\begin{abstract}
The classical works in finance and insurance for modeling asset returns is the Gaussian model. However, when modeling complex random phenomena, more flexible distributions are needed which are beyond the normal distribution. This is because most of the financial and economic data are skewed and have "fat tails". Hence symmetric distributions like normal or others may not be good choices while modeling these kinds of data. Flexible distributions like skew normal distribution allow robust modeling of high-dimensional multimodal and asymmetric data. In this paper, we consider a very flexible financial model to construct comonotonic lower convex order bounds in approximating the distribution of the sums of dependent log skew normal random variables. The dependence structure of these random variables is based on a recently developed generalized multivariate skew normal distribution, known as unified skew normal distribution. The approximations are used to calculate the risk measure related to the distribution of terminal wealth. The accurateness of the approximation is investigated numerically. Results obtained from our methods are competitive with a more time consuming method known as Monte Carlo method.
\end{abstract}

Keywords and phrases: Unified skew normal distribution, additive properties, log unified skew normal distribution, convex order, comonotonicity, value at risk.

\section{Introduction}

In this paper we investigate the approximations for the distribution function of a sum of log skew normal random variables. Let $\alpha 0, \alpha 1, \alpha 2, \ldots, \alpha \mathrm{n}-1$ be non-negative real numbers and $\mathrm{Y}=(\mathrm{Y} 1, \mathrm{Y} 2, \ldots, \mathrm{Yn}) \mathrm{T}$ be a multivariate skew normal random vector with the specified mean vectorand variance-covariance matrix and satisfying additive properties. Define $Z_{i}=\sum_{k=i+1}^{n} Y_{k}, i=0,1, \ldots, n-1$, that is, $Z_{i}^{\prime}$ s are sums of the components $\left(Y_{1}, Y_{2}, \ldots, Y_{n}\right)$. With the components so defined, consider the sum

$$
S=\sum_{i=0}^{n-1} \alpha_{i} e^{Z_{i}}=\sum_{i=0}^{n-1} \alpha_{i} e^{Y_{i+1}+\cdots+Y_{n}}
$$

From economic or actuarial point of view, the sum S could be interpreted as the final wealth or the terminal wealth or the accumulated value of a series of deterministic saving amounts or alternatively the accumulated value of a

\footnotetext{
*Corresponding Author, email:gupta@bgsu.edu
} 
series of payments. In this situation, $\alpha \mathrm{i}(\mathrm{i}=0, \ldots, \mathrm{n}-1)$ represents yearly saving in period $\mathrm{i}$ or amount invested in period $\mathrm{i}, \mathrm{Yi}+1$ refers to the random rate of return in period $\mathrm{i}$ for $\mathrm{i}=0, \ldots, \mathrm{n}-1$. The term $Y_{k}=\log \frac{P_{k}}{P_{k-1}}=\log P_{k}-\log P_{k-1}$ i.e $e^{Y_{k}}=\frac{P_{k}}{P_{k-1}}$, where $\mathrm{Pk}$ is the price of the asset at the period $\mathrm{k}=0, \ldots, \mathrm{n} ;$ is called the random log-return in period $\mathrm{k}$ and $\mathrm{Zi}$ denote the sum of stochastic or random returns in the period $\mathrm{i}=$ $0, \ldots, n-1$. With some suitable adjustment, $S$ could also be referred as the present value of a series of payments. More precisely, if $-\mathrm{Zi}$ denotes the stochastic log-return over the period [0,i], then eZi represents the stochastic discount factor over the period $[0, \mathrm{i}]$. In this situation, the sum $\mathrm{S}$ is the present value of $\alpha \mathrm{i}$ (Vanduffel et al. 2008, Roch and Valdez (2011)).

The sum defined in (1.1) plays a central role in the actuarial and financial theory because it allows computation of risk measures such as value at risk or stop-loss premium. To calculate the risk measures we need to evaluate the distribution function of S. Unfortunately, the distribution of the sum S (of log-normally or log-skew normally distributed random variables) is not available in closed-form. It is possible to use Monte Carlo simulation method to numerically approximate the distribution function. However, Monte Carlo simulation of the distribution is often timeconsuming. Thus one has to find alternative way to approximate the distribution of the sum. Among the proposed solutions, moment matching methods and inverse gamma approximations are commonly used. Both methods approximate the unknown distribution function by a given one such that the first two moments coincide.

Kaas et al. (2001) and Dhaene et al. ( 2002a, 2002b) propose to approximate the distribution function of S by so called "convex lower bound". The underlying idea of convex lower order bound is to replace an unknown or too complex distribution (for which no explicit form is found) by another one which is easier to determine. In this approach, the actual distribution is known to be bounded in terms of convex ordering to the approximated distribution. To be more precise, the distribution function of $S=\sum_{i=0}^{n-1} \alpha_{i} e^{Z_{i}}$ is approximated by the distribution function of Sl, where $\mathrm{Sl}$ is defined by,

$$
\mathrm{S}_{l}=\sum_{i=0}^{n-1} \alpha_{i} E\left(e^{Z_{i}} \mid \Lambda\right)
$$

An appropriate choice of the conditioning random variable $\Lambda$ is required. This approach has twofold advantages. Firstly, use of this approach transforms the multidimensional problem caused by $(\mathrm{Z} 0, \mathrm{Z} 2, \ldots, \mathrm{Zn}-1)$ to a single dimension caused by $\Lambda$. Secondly, an appropriate choice of $\Lambda$ (that makes the expectation in (1.2) nondecreasing or non-increasing function of the conditioning random variable $\Lambda$ ) will make a comonotonic sum, i.e, the elements of the sum in (1.2) possess the so called comonotonic dependence structure. Using the additivity properties of sum of comonotonic random variables, risk measure related to the distribution function of $\mathrm{S}$ is then approximated by the corresponding risk measure of Sl. According to Kaas et al. (2001), comonotonic upper bound for the sum in convex order sense can also be derived using the result

$$
\sum_{i=0}^{n-1} X_{i} \leq \sum_{i=0}^{n-1} F_{X_{i}}(U)
$$

where $\mathrm{U}$ is the uniform random variable over $(0,1)$. However, the comonotonic upper bounds generally provide too conservative estimates of the cumulative distribution function (Roch and Valdez 2011). Thus we only discuss convex lower bound here. The model given in (1.1) was studued by many authors as referred above. Our work in this paper is the natural extension of the work by Marin-Solano et al. (2010), and Roch and Valdez (2011).

The rest of the paper is organized as follows. In Section 2 we present some basic properties of the unified skew normal (SUN) density such as the moment generating function, mean and variance, and establish the 
additive stability. Section 3 provides basic concepts and important result on convex ordering and convex order bounds. Section 4 discusses in detail the construction of lower convex order bounds for the distribution of sum of $\log$ unified skew normal random variables. Section 5 provides an extension of section 4 considering the construction of lower bounds for a portfolio. Numerical illustrations are provided in Section 6. The paper concludes in Section 7 in which some discussions and suggestions are provided.

\section{The unified skew normal distribution and its important properties}

Skew normal class of distributions is a natural extension of normal distribution which is developed to include the skewness of data. This class of distributions has properties that resemble to those of the normal distribution. In this paper, we consider the multivariate unified skew normal distribution introduced by Arellano-Valle and Azzalini (2006). In addition to being normal when the skewness parameter equals zero, the family has properties similar to the normal distribution and yet is skew.

Suppose $\varphi \mathrm{d}(\mathrm{y}-\mu ; \Omega)$ denotes the d dimensional multivariate normal density with mean vector $\mu$ and the covariance matrix $\Omega$, and $\Phi \mathrm{d}(\mathrm{y}-\mu ; \Omega)$ is the corresponding distribution function.

Definition 2.1. (Arellano-Valle and Azzalini (2006)) A random vector $y \in<\mathrm{d}$ is said to follow unified skew normal distribution, if its density function is given by

$$
f(y)=\varphi_{d}(\mathrm{y}-\mu ; \Omega) \frac{\Phi m\left(\gamma+\Delta T \Omega^{-}-1 \omega-1(y-\mu) ; \Gamma-\Delta T \Omega^{-}-1 \Delta\right)}{\Phi m(\gamma ; \Gamma)}
$$

where $\mu \in \Re^{d}, \gamma \in \Re^{m}, \Omega \in \Re^{d \times d}, \Gamma \in \Re^{m \times m}, \Delta \in \Re^{d \times m}$ and $\Omega^{*}=\left(\begin{array}{cc}\Gamma & \Delta^{T} \\ \Delta & \bar{\Omega}\end{array}\right)$ is the correlation matrix with $\omega$ a $d \times d$ diagonal matrix formed by the standard deviations of $\Omega$; hence $\Omega=\omega \Omega^{-} \omega$.

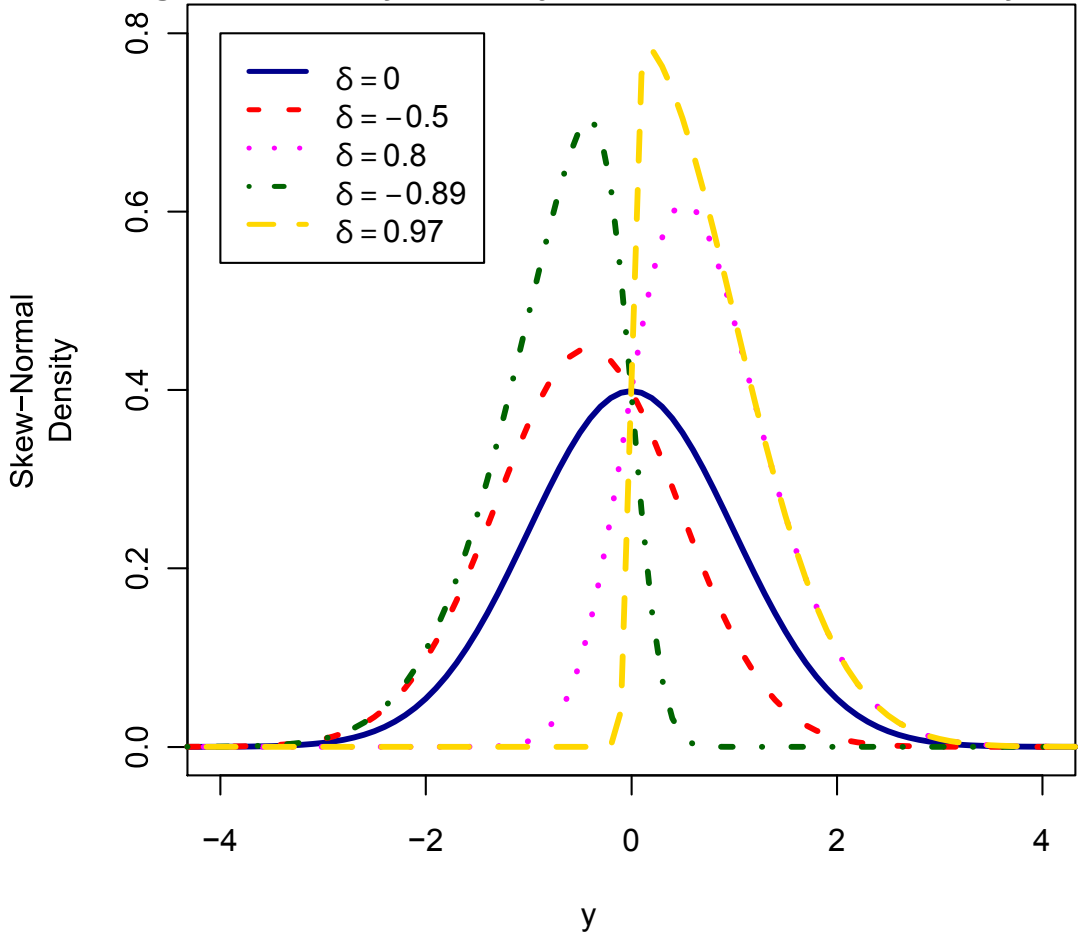


Figure 1: Univariate SUN density for somme selected values of $\delta$.

The density is called SUN (acronym for unified skew normal distribution) and is denoted by $\operatorname{SUNd}, \mathrm{m}(\mu, \gamma, \bar{\omega}, \Omega *)$, where $\omega^{-}=\omega 1 \mathrm{~d}$ is the vector of diagonal elements of $\omega$. Note that if $\Delta$ equal to zero, then the density reduces to the dimensional multivariate normal distribution.

The corresponding distribution function of $y$ is given by,

$$
G_{d, m}(\boldsymbol{y})=\frac{1}{\Phi_{m}(\boldsymbol{\gamma} ; \Gamma)} \Phi_{d+m}\left[\left(\begin{array}{l}
\boldsymbol{y} \\
\mathbf{0}
\end{array}\right) ;\left(\begin{array}{c}
\boldsymbol{\mu} \\
-\boldsymbol{\gamma}
\end{array}\right),\left(\begin{array}{cc}
\Omega & -\omega \Delta \\
-\Delta^{T} \omega & \Gamma
\end{array}\right)\right]
$$

The derivation of the SUN density was given in Arellano-Valle and Azzalini (2006). The graph of the univariate SUN density is given in Figure 1 for different values of the skewness parameter $\delta$.

One of the advantages of parameterization in (2.1) resides in the additive stability of the distribution. Gupta et al. (2013) proved the closure properties of independent SUN random vectors: closure under linear transformations, marginal and conditional distributions and joint distribution from its members. In what follows, we will state some of these important properties. For more details and the proofs of these results, we refer to Gupta et al. (2013) and Arellano-Valle and Azzalini (2006).

Theorem 2.1. If $\mathrm{y} \sim \mathrm{SUNd}, \mathrm{m}\left(\mu, \gamma, \omega^{-}, \Omega *\right)$, then its m.g.f is given by

$$
M y(t)=\exp \left(\mu^{T} t+\frac{1}{2} t^{T} \Omega t\right) \frac{\Phi m(\gamma+\Delta T \omega t ; \Gamma)}{\Phi_{m}(\gamma ; \Gamma)}, t \in R^{d}
$$

Theorem 2.2. If $\mathrm{y} \sim \mathrm{SUNd}, \mathrm{m}\left(\mu, \gamma, \omega^{-}, \Omega *\right)$, then the mean vector and the covariance matrix of $\mathrm{y}$ are given by

And

$$
\mathrm{E}(\mathrm{y})=\mu+\frac{\Phi_{m}{ }^{*}(\gamma ; \Gamma)}{\Phi_{m}(\gamma ; \Gamma)} \Phi_{m}(\gamma ; \Gamma)
$$

$$
\operatorname{Var}(y)=\Omega+\frac{\Phi_{m}{ }^{* *}(\gamma ; \Gamma)}{\Phi_{m}(\gamma ; \Gamma)}-\frac{\Phi_{m}{ }^{*}(\gamma ; \Gamma)}{\Phi_{m}(\gamma ; \Gamma)} \frac{\Phi_{m}{ }^{* T}(\gamma ; \Gamma)}{\Phi_{m}(\gamma ; \Gamma)}
$$



Using Lemma B.1 given in the Appendix of Domingnez-Monila et al. (2001) the term $\Phi_{m}^{*}(\gamma ; \Gamma)$ could be replaced by,

$$
\Phi_{m}^{*}(\gamma ; \Gamma)=\sum_{i=1}^{d} \sum_{j=1}^{m}\left(\Delta^{T} \omega\right)_{i j} \Phi_{m}^{\{j\}}(\gamma ; \Gamma) e_{i}
$$


where $\left(\Delta^{T} \omega\right)_{i j}$ is the $(i, j)$ th element of the matrix $\Delta^{T} \omega, e_{i}$ is a $d \times 1$ vector with one in the $i$ th position and zero elsewhere, and

$$
\Phi_{m}^{\{j\}}(\gamma ; \Gamma)=\varphi\left(\gamma_{j} ; \Gamma_{i j}\right) \Phi_{m-1}\left(\gamma_{-j} ; \Gamma \mid \gamma_{j}\right)
$$

where $\gamma$-j is the vector $\gamma$ without the $j$ th element.

Corollary 2.1. Let y be a random vector with a unified skew normal distribution, $\operatorname{SUN}_{\mathrm{d}, \mathrm{m}}\left(\mu, \mathbf{0}, \omega^{-}, \Omega^{*}\right)$, with $\Omega^{*}=\left(\begin{array}{cc}I_{m} & \Delta^{T} \\ \Delta & \bar{\Omega}\end{array}\right)$. Then the first two moments of $\mathrm{y}$ are:

$$
\begin{aligned}
& \text { (a) } \mathbb{E}(\boldsymbol{y})=\boldsymbol{\mu}+\sqrt{\frac{2}{\pi}} \omega \Delta \mathbf{1}_{m} \\
& \text { (b) } \operatorname{Var}(\boldsymbol{y})=\Omega-\frac{2}{\pi} \omega \Delta \Delta^{T} \omega
\end{aligned}
$$

Example 2.1. Suppose $Y \sim S U N_{1,1}\left(\mu, 0, w, \Omega^{*}\right)$ with $\Omega^{*}=\left(\begin{array}{cc}g^{2} & \delta \\ \delta & v\end{array}\right)$. Then

$$
f(y)=2 \varphi\left(y ; \mu, \sigma^{2}\right) \Phi\left(\delta v^{-1} w^{-1}(y-\mu) ; g^{2}-\delta^{2} / v^{-1}\right)
$$

- the mean of this density is

- and the variance is

$$
\mathbb{E} y=\mu+2 \Phi_{1}^{*}\left(0 ; g^{2}\right)=\mu+\frac{\delta w}{g} \sqrt{\frac{2}{\pi}}
$$

$$
\operatorname{Var}(y)=\sigma^{2}+2 \Phi_{1}^{* *}\left(0 ; g^{2}\right)-\left(2 \Phi_{1}^{*}\left(0 ; g^{2}\right)\right)^{2}=\sigma^{2}-\frac{2}{\pi} \frac{\delta^{2} w^{2}}{g^{2}}
$$

One of the most important additive properties of the SUN random vector is the closure under the linear transformation property. Similar to the multivariate normal distribution, SUN distribution is closed under full row (or column) rank linear transformation. This property is useful to establish joint distribution of the independent random variables from the same family.

Theorem 2.3. Let $\mathrm{y} \sim \operatorname{SUN}_{\mathrm{d}, \mathrm{m}}\left(\mu, \gamma, \omega^{-}, \Omega^{*}\right)$ and $\mathrm{A}$ be an $\mathrm{n} \times \mathrm{d}(\mathrm{n} \leq \mathrm{d})$ matrix of rank $\mathrm{n}$. Then

$$
A \boldsymbol{y} \sim S U N_{n, m}\left(\boldsymbol{\mu}_{A}, \boldsymbol{\gamma}, \bar{\omega}_{A}, \Omega_{A}^{*}\right)
$$

where

$$
\begin{gathered}
\mu_{A}=A \boldsymbol{\mu}, \quad \overline{\boldsymbol{\omega}}_{A}=\omega_{A} 1_{n}, \quad \omega_{A}=A \omega A^{T} \quad \Omega_{A}^{*}=\left(\begin{array}{cc}
\Gamma & \Delta_{A}^{T} \\
\Delta_{A} & \bar{\Omega}_{A}
\end{array}\right), \quad \Delta_{A}=\left(A \omega A^{T}\right)^{-1} A \omega \Delta \\
\Omega_{A}^{-}=(A \omega A T)-1 A \Omega A T(A \omega A T)-1 \quad \text { and } \Omega_{A}=A \Omega A T=\omega A \Omega^{-}{ }_{A} \omega A .
\end{gathered}
$$

As previously mentioned, we can easily build the joint distribution of independent SUN random variables which again belongs to the same family. In the following Theorem, we will show that if we have a collection 
of $\mathrm{n}$ independent SUN random variables, then the joint distribution of the $\mathrm{n}$ random variables is again SUN distributed random variable. As mentioned by Gupta et al. (2004), this property does not hold for the multivariate skew normal distribution defined by Azzalini and Dalla Valle (1996).

In what follows the symbols " $\otimes$ " and " $\oplus$ " would be used to indicate the Kronecker matrix product and the matrix direct sum operator: the Kronecker product for any two matrices $A m \times n=$

(aij) and $\mathrm{Bp} \times \mathrm{q}=($ bij) denoted by $\mathrm{A} \otimes \mathrm{B}$ results an $\mathrm{mp} \times$ nq matrix defined by

$$
A \otimes B=\left[\begin{array}{cc}
a_{11} B & a_{12} B \ldots a_{1 n} B \\
a_{21} B & a_{22} B \ldots a_{2 n} B \\
\vdots & \\
a_{m 1} B & a_{m 2} B \ldots a_{m n} B
\end{array}\right]=\left(a_{i j} B\right)
$$

For the properties of the Kronecker matrix product we refer to Gupta and Nagar (2000).

The direct sum operator of two matrices $A m \times n=($ aij $)$ and $B p \times q=($ bij) denoted by $A \oplus B$ results an $(m$ $+\mathrm{p}) \times(\mathrm{n}+\mathrm{q})$ matrix defined by

$$
\left[\begin{array}{ll}
A & 0 \\
0 & B
\end{array}\right]
$$

That is matrix direct sum operator gives a block diagonal matrix.

Note: It is easy to see that $\oplus_{i=1}^{n} A=I_{n} \otimes A$. For other properties of matrix direct sum operator we refer to Horn and Johnson (1991).

Theorem 2.4. Suppose $y_{1}, \ldots, y_{n}$ are independent random vectors with $y_{i}$ ? $S U N_{d i}, m_{i}\left(\mu_{i}, \gamma_{i}, \omega_{i}^{-}, \Omega_{i}^{\square}\right)$.

Then the joint distribution of $y_{1}, \ldots, y_{n}$ is given by

$$
\boldsymbol{y}=\left(\boldsymbol{y}_{1}^{T}, \ldots \boldsymbol{y}_{n}^{T}\right)^{T} \sim S U N_{d^{\dagger}, m^{\dagger}}\left(\boldsymbol{\mu}^{\dagger}, \boldsymbol{\gamma}^{\dagger}, \overline{\boldsymbol{\omega}}^{\dagger}, \Omega^{* \dagger}\right)
$$

where

$$
d_{\dagger}=\sum_{i=1}^{n} \mathrm{~d}_{i}, \quad m_{\dagger}=\sum_{i=1}^{n} \mathrm{X} m_{i}, \mu \dagger=\left(\mu_{1}^{T}, \ldots \mu_{n}^{T}\right)^{T}, \gamma_{\dagger}=\left(\gamma_{1}^{T}, \ldots \gamma_{n}^{T}\right)_{T}, \bar{\omega}_{\dagger}=\left(\bar{\omega}_{1}^{T}, \ldots \bar{\omega}_{n}^{T}\right)^{T}
$$

and

$$
\omega^{\dagger}=\oplus_{i=1}^{n} \omega_{i} \quad \Omega^{\dagger}=\oplus_{i=1}^{n} \Omega_{i}, \quad \bar{\Omega}^{\dagger}=\oplus_{i=1}^{n} \bar{\Omega}_{i}, \quad \Gamma^{\dagger}=\oplus_{i=1}^{n} \Gamma_{i}, \quad \Delta^{\dagger}=\oplus_{i=1}^{n} \Delta_{i}, \quad \Omega^{* \dagger}=\left(\begin{array}{cc}
\Gamma^{\dagger} & \Delta^{\dagger^{T}} \\
\Delta^{\dagger} & \bar{\Omega}^{\dagger}
\end{array}\right)
$$

As we have seen, in the previous theorem the SUN random vectors need not to be identically distributed. Addition of identical assumption leads to the following corollary.

Corollary 2.2. If $\mathrm{y}_{1}, \ldots, \mathrm{y}_{\mathrm{n}}$ are independent and identically distributed (iid) random vectors from the $\operatorname{SUN}_{\mathrm{d}, \mathrm{m}}\left(\mu, \gamma, \omega^{-}, \Omega^{*}\right)$ distribution, then the joint distribution of $\mathrm{y}_{1}, \ldots, \mathrm{y}_{\mathrm{n}}$ is

$$
\boldsymbol{y}=\left(\boldsymbol{y}_{1}^{T}, \ldots \boldsymbol{y}_{n}^{T}\right)^{T} \sim S U N_{d^{\dagger}, m^{\dagger}}\left(\boldsymbol{\mu}^{\dagger}, \boldsymbol{\gamma}^{\dagger}, \overline{\boldsymbol{\omega}}^{\dagger}, \Omega^{* \dagger}\right)
$$

where

$$
d^{\dagger}=n d, \quad m^{\dagger}=n m, \mu^{\dagger}=\mathbf{1}_{n} \otimes \mu, \gamma^{\dagger}=\mathbf{1}_{n} \otimes \gamma, \omega^{-\dagger}=\mathbf{1}_{n} \otimes \omega^{-},
$$


and

$$
\Omega^{\dagger}=I_{n} \otimes \Omega, \quad \bar{\Omega}^{\dagger}=I_{n} \otimes \bar{\Omega}, \quad \Delta^{\dagger}=I_{n} \otimes \Delta, \quad \Gamma^{\dagger}=I_{n} \otimes \Gamma, \quad \Omega^{* \dagger}=\left(\begin{array}{cc}
\Gamma^{\dagger} & \Delta^{\dagger^{T}} \\
\Delta^{\dagger} & \bar{\Omega}^{\dagger}
\end{array}\right)
$$

Another interesting property of the SUN family is the closure under the marginalization and conditional distributions. These results are stated in the following theorems:

Theorem 2.5. Let y be a random vector distributed as $\operatorname{SUN}_{\mathrm{d}, \mathrm{m}}\left(\mu, \gamma, \omega^{-}, \Omega^{*}\right)$ and be partitioned as $y=\left(\begin{array}{l}\boldsymbol{y}_{1} \\ \boldsymbol{y}_{2}\end{array}\right)_{d-k} k$. Consider a $k \times d$ matrix $A=\left(I_{k} 0\right)$, with a $k \times k$ identity matrix $I_{k}$ and $a k \times(d-k)$ zero matrix 0. Then the marginal distribution of $y_{1}=$ Ay is $S U N_{k, m}\left(\boldsymbol{\mu}_{1}, \boldsymbol{\gamma}, \bar{\omega}_{1}, \Omega_{1}^{*}\right)$,

where $\quad \boldsymbol{\mu}=\left(\begin{array}{l}\boldsymbol{\mu}_{1} \\ \boldsymbol{\mu}_{2}\end{array}\right)_{d-k}^{k}, \quad \overline{\boldsymbol{\omega}}=\left(\begin{array}{c}\overline{\boldsymbol{\omega}}_{1} \\ \overline{\boldsymbol{\omega}}_{2}\end{array}\right)_{d-k}^{k}, \Delta=\left(\begin{array}{c}\Delta_{1} \\ \Delta_{2}\end{array}\right)_{d-k}^{k}$

$$
\Omega_{1}^{*}=\left(\begin{array}{cc}
\Gamma & \Delta_{1}^{T} \\
\Delta_{1} & \bar{\Omega}_{11}
\end{array}\right) \text { and } \bar{\Omega}=\left(\begin{array}{cc}
\bar{\Omega}_{11} & \bar{\Omega}_{12} \\
\bar{\Omega}_{21} & \bar{\Omega}_{22}
\end{array}\right) d-k
$$

Theorem 2.6. Let y be a random vector distributed as y $\sim \operatorname{SUN}_{\mathrm{d}, \mathrm{m}}\left(\mu, \gamma, \omega^{-}, \Omega^{*}\right)$. Consider two subvectors $\mathrm{y}_{1}$ and $\mathrm{y}_{2}$, where $\mathrm{y}^{T}=\left(\begin{array}{ll}\boldsymbol{y}_{1}^{T} & \boldsymbol{y}_{2}^{T}\end{array}\right), \boldsymbol{y}_{1}$ is $k$ dimensional. Suppose $\mu, \omega,{ }^{-} \Delta$ and $\Omega^{-}$are partitioned as in above Theorem. Then the conditional distribution of $\mathrm{y}_{1}$ given $\mathrm{y}_{2}=\mathrm{y}_{\mathrm{z} 0}$ is $S U N_{k, m}\left(\boldsymbol{\mu}_{1.2}, \gamma_{1.2}, \bar{\omega}_{1}, \Omega_{11.2}^{*}\right)$, where

$$
\begin{gathered}
\mu 1.2=\boldsymbol{\mu}_{1}+\Omega_{12} \Omega_{22}^{-1}\left(y_{10}-\boldsymbol{\mu}_{2}\right), \gamma_{1.2}=\gamma+\Delta_{2}^{T} \bar{\Omega}_{22}^{-1} \omega_{2}^{-1}\left(y_{z 0}-\boldsymbol{\mu}_{2}\right), \bar{\Omega}_{11.2}=\bar{\Omega}_{11}-\bar{\Omega}_{12} \bar{\Omega}_{22}^{-1} \bar{\Omega}_{21}, \\
\Omega_{11.2}=\omega_{1} \bar{\Omega}_{11.2} \omega_{1}=\Omega_{11}-\Omega_{12} \Omega_{22}^{-1} \Omega_{21} \text { with } \Omega_{i j}=\omega_{i} \bar{\Omega}_{i j} \omega_{j}, i, j=1,2, \Delta_{1.2}=\Delta_{1}-\bar{\Omega}_{12} \bar{\Omega}_{22}^{-1} \Delta_{2}, \\
\Gamma_{1.2}=\Gamma-\Delta_{2}^{T} \bar{\Omega}_{22}^{-1} \Delta_{2} \text { and } \Omega_{1.2}^{*}=\left(\begin{array}{cc}
\Gamma_{1.2} & \Delta_{1.2}^{T} \\
\Delta_{1.2} & \bar{\Omega}_{11.2}
\end{array}\right) .
\end{gathered}
$$

\section{Convex order and convex order bounds}

Definition 3.1. Consider two random variables $X$ and $Y$ such that $E[\varphi(X)] \leq E[\varphi(Y)]$, for all the convex functions $\varphi$, provided expectations exist. Then $X$ is said to be smaller than $Y$ in the convex order denoted as $X \leq_{c x} Y$.

We state an important result related to the convex ordering of random variables in the following lemma. We need this result to evaluate the lower bounds for the distribution of sum of log unified skew normal density.

Lemma 3.1. (Dhaene et al. (2002b)) For any random vector $X=\left(X_{1}, X_{2}, \ldots, X_{n}\right)^{T}$ and any random variable $\Lambda$, which is assumed to be a function of $X$, we have,

$$
\sum_{i=1}^{n} \mathbb{E}\left[X_{i} \mid \Lambda\right] \leq_{c x} \sum_{i=1}^{n} X_{i}
$$


According to this result, we can approximate the sum $S$ in (1.1) by the sum $S 1$ in (1.2). However, as mentioned in section 1, an appropriate choice of $\Lambda$ is required as different choices of $\Lambda$ lead to different approximations. Interesting choices of $\Lambda$ are those that make the sum Sl a comonotonic sum. We use an important property of comonotonic sum to compute risk measure in section 6. Detailed information about convex order bounds and comonotonicity can be found in Dhaene et al. (2002a, 2002b).

Besides computing lower bounds for the terminal wealth, as an application we discuss the evaluation of risk measures of S. A risk measure provides the information contained in the distribution function of a random variable in one single real number. One of the most commonly used risk measures in the field of actuarial science and financial economics is the p-quantile risk measure, based on a percentile concept known as valueat-risk (VaR). In probabilistic terms, the $\mathrm{VaR}$ at level $\mathrm{p}$ is defined as the $100 \mathrm{p} \%$ quantile of the distribution of the terminal wealth. More precisely, for any $p \in(0,1)$, the $p$-quantile measure or $\mathrm{VaR}$ for a random variable $\mathrm{X}$, denoted by $\mathrm{Qp}[\mathrm{X}]$, is defined as

$$
Q_{p}[X]=\inf \left\{x \in<\mid F_{X}(x) \geq p\right\}
$$

When the marginal risks possess the comonotonic dependence structure, the global value-atrisk can be obtained by summing up the marginal VaR measures. Thus for a comonotonic random vector $\mathrm{X}=$ $(\mathrm{X} 1, \mathrm{X} 2, \ldots, \mathrm{Xn}) \mathrm{T}$ and the $\operatorname{sum} S=\sum_{i=1}^{n} X_{i}$, the value at risk $(\mathrm{VaR})$ is

$$
Q_{P}(S)=\sum_{i=1}^{n} Q P[X i]
$$

\section{Distribution of sum of $\log$ SUN random varibles}

In this section we derive the bounds to approximate the distribution of sums of log unified skew normal variables. The bounds that we present here are natural extensions to the results obtained by Marin-Solano et al. (2010) and Roch and Valdez (2011). The derivation of this bound requires some results that are presented in the following lemmas. Recall that $\mathrm{Yk}$ denotes the random logreturn in the period $\mathrm{k}$, for $\mathrm{k}=1,2, \ldots, \mathrm{n}$ and $\mathrm{Zi}$ denotes the accumulated returns from the time i to the final time $\mathrm{t}=\mathrm{n}$.

Lemma 4.1. (Joint distribution of $\left.Y=\left(Y_{1}, \ldots, Y_{n}\right)^{T}\right)$ Let $Y_{k}, k=1, \ldots, n$ be univariate iid random variables distributed as

$$
\operatorname{SUN}_{1, m}\left(\mu, \gamma, \bar{\omega}, \Omega^{*}\right), \text { where } \Omega^{*}=\left(\begin{array}{cc}
\Gamma & \Delta^{T} \\
\Delta & \bar{\Omega}
\end{array}\right)
$$

Then the distribution of $Y=\left(Y_{1}, \ldots, Y_{n}\right)^{T}$ is

$$
\operatorname{SUNn}, m n\left(\mu_{Y}, \gamma_{Y}, \bar{\omega}_{Y}, \Omega_{Y}^{*}\right)
$$

where

$$
\mu_{Y}=\mathbf{1}_{n} \otimes \mu, \gamma_{Y}=\mathbf{1}_{n} \otimes \gamma, \omega_{Y}=I_{n} \otimes \omega \omega_{Y}^{-}=\omega_{Y} \otimes \mathbf{1}_{n}
$$

and

$$
\Omega_{Y}=I_{n} \otimes \Omega, \quad \bar{\Omega}_{Y}=I_{n} \otimes \bar{\Omega}, \quad \Delta_{Y}=I_{n} \otimes \Delta, \quad \Gamma_{Y}=I_{n} \otimes \Gamma, \quad \Omega_{Y}^{*}=\left(\begin{array}{cc}
\Gamma_{Y} & \Delta_{Y}^{T} \\
\Delta_{Y} & \bar{\Omega}_{Y}
\end{array}\right)
$$


Proof. The proof follows from Theorem 2.2 by noting that $\oplus_{1}^{n}=I_{n} \otimes A$ for any matrix $A$ where $I_{n}$ is an $n \times n$ identity matrix and $\mathbf{1}_{n}$ is a unit vector of dimension $n$.

Lemma 4.2. (Joint distribution of $\left.Z=\left(Z_{0}, \ldots, Z_{n-1}\right)^{T}\right)$ Let $Z_{i}, i=0, \ldots, n-1$ be the sum of returns of one unit of capital invested from time $t=i$ to the final time $t=n$, that is, $Z_{i}=\sum_{k=i+1}^{n} Y_{k^{*}}$ Let $T \in<^{n \times n}$ be an upper unit triangular matrix. Then the distribution of $Z=\left(Z_{0}, . ., Z_{n-1}\right)^{T}$ is

$$
S U N_{n, m n}\left(\mu_{Z}, \gamma_{Z}, \bar{\omega}_{Z}, \Omega_{Z}^{*}\right)
$$

where

$$
\mu_{Z}=T \boldsymbol{\mu}_{Y}, \quad \gamma_{Z}=\gamma_{Y}, \quad \omega_{Z}=T \omega_{Y} T^{T}, \quad \overline{\boldsymbol{\omega}}_{Z}=\omega_{Z} \mathbf{1}_{n}
$$

and

$$
\Omega_{Z}=T \Omega_{Y} T^{T}, \quad \bar{\Omega}_{Z}=\omega_{Z}^{-1} \Omega_{Z} \omega_{Z}^{-1}, \quad \Delta_{Z}=\left(T \Omega_{Y} T^{T}\right)^{-1} T \omega_{Y} \Delta_{Y}, \quad \Gamma_{Z}=\Gamma_{Y}, \quad \Omega_{Z}^{*}=\left(\begin{array}{cc}
\Gamma_{Z} & \Delta_{Z}^{T} \\
\Delta_{Z} & \bar{\Omega}_{Z}
\end{array}\right)
$$

Proof. The proof follows from Theorem 2.3 with $T$ being the matrix of coefficients.

As mentioned in Section 1, the comonotonicity of the convex lower bound strongly depends on the special choice of the conditioning random variable $\Lambda$. Therefore, it is required to choose a functional form of this random variable. Since a good choice of $\Lambda$ is important in determining the accurate approximations for the final wealth, different choices of $\Lambda$ have been proposed in the literature. Following Dhaene et al. (2002a), we will choose $\Lambda$ in such a way that it becomes a linear transformation of a first order approximation to Sn. This is known as "Taylor-based" approach. In this approach, $\Lambda$ is defined as,

$$
\Lambda=\sum_{i=0}^{n-1} v_{i} Z_{i}
$$

with the choice of the coefficents $v_{i}$, as $v_{i}=\alpha_{i} e^{\mathrm{E}[Z i]}$. If the random variables $Y_{k}, k=1, \ldots, n$ are iid then the coefficients $v_{i}$ is given by $v_{i}=\alpha_{i} e^{\mathrm{E}[Z i]}=\alpha_{i} e^{\mathrm{E}[Y]}$.

Lemma 4.3. (Distribution of $\Lambda$ ) Let the random variable $\Lambda$ be defined by $\Lambda=\sum_{i=0}^{n-1} \nu_{i} Z_{i}$ and $V=\left(v_{0}, \ldots v_{n-1}\right)$ be a row vector. Then the distribution of $\Lambda$ is

$$
S U N_{1, m n}\left(\mu_{\Lambda}, \gamma_{\Lambda}, \bar{\omega}_{\Lambda}, \Omega_{\Lambda}^{*}\right)
$$

where

$$
\mu_{\Lambda}=V \mu_{Z}, \quad \gamma_{\Lambda}=\gamma_{Z}, \quad \omega_{\Lambda}=V \omega_{Z} \boldsymbol{V}^{t}, \quad \bar{\omega}_{\Lambda}=\omega_{\Lambda} 1
$$

and



Proof. The proof follows from the Theorem 2.3 with $V$ being the vector of coefficients.

Lemma 4.4. (Joint distribution of $\Lambda$ and each of the elements of vector Z) Let $S_{i} \in<^{2 \times n}$ be a matrix with the first row as $V$ and second row of 0 's except in column $i+1$ where the 0 is replaced by 1 . That is 


$$
M_{i}=\left(\begin{array}{ccccc}
\nu_{0} & \nu_{1} & \ldots & \nu_{i} \ldots & \nu_{n-1} \\
0 & 0 & \ldots & 1 \ldots & 0
\end{array}\right)
$$

Then the distribution of $\left.X_{i}=\Lambda, Z_{i}\right)^{T}$ is

$$
S U N_{2, m n}\left(\boldsymbol{\mu}_{X_{i}}, \gamma_{X_{i}}, \bar{\omega}_{X_{i}}, \Omega_{X_{i}}^{*}\right)
$$

where

$$
\begin{gathered}
\mu_{X_{i}}=M_{i} \boldsymbol{\mu}_{Z}, \quad \gamma_{X_{i}}=\gamma_{Z}, \quad \omega_{X_{i}}=M_{i} \omega_{Z} M_{i}^{T}, \quad \overline{\boldsymbol{\omega}}_{X_{i}}=\omega_{X_{i}} \mathbf{1}_{2} \\
\Omega_{X_{i}}=M_{i} \Omega_{Z} S_{i}^{T}, \quad \bar{\Omega}_{X_{i}}=\omega_{X_{i}}^{-1} \Omega_{X_{i}} \omega_{X_{i}}^{-1}, \quad \Delta_{X_{i}}=\left(M_{i} \omega_{X_{i}} M_{i}^{T}\right)^{-1} M_{i} \omega_{X_{i}} \Delta_{Z}, \quad \Gamma_{X_{i}}=\Gamma_{Z},
\end{gathered}
$$

and

$$
\Omega_{X_{i}}^{*}=\left(\begin{array}{cc}
\Gamma_{X_{i}} & \Delta_{X_{i}}^{T} \\
\Delta_{X_{i}} & \bar{\Omega}_{X_{i}}
\end{array}\right)
$$

Proof. The proof follows from Theorem 2.3 with $M_{i}$ being the matrix of coefficients.

Lemma 4.5. (Conditional distribution of $Z_{i} \mid \Lambda=\lambda$ ) Let $\mu_{X i},{ }^{\Omega^{-}}{ }_{X i}, \omega^{-}{ }_{X i}$ and $\Delta_{X i}$ be partitioned as in Theorem 2.5. Then the distribution of $H_{i}=\left(Z_{i} \mid \Lambda=\lambda\right)$ is given by

$$
S U N 1, m n\left(\mu H_{i}, \gamma_{i}, \omega^{-} H_{i}, \Omega * H_{i}\right),
$$

where

$$
\mu H_{i}=\mu 2+\Omega_{21} \Omega-211\left(\lambda-\mu_{1}\right), \gamma_{H i}=\gamma X_{i}+\Delta T 1 \Omega^{-}-111 \omega 2-1\left(\lambda-\mu_{1}\right), \omega^{-} H_{i}=\omega^{-} 1,
$$

$$
\Gamma_{H_{i}}=\Gamma X_{i}-\Delta T 1 \Omega^{-}{ }_{-111} \Delta 1, \Delta H_{i}=\Delta 2-\Omega 21^{-} \Omega^{-}{ }_{11-1} \Delta 1, \Omega_{H_{i}}^{-}=\Omega^{-} 22-\Omega_{21}^{-} \Omega^{-}{ }_{11-1} \Omega_{12}^{-}, \quad \Omega_{H_{i}}=\omega_{H_{i}} \Omega^{-}{ }_{H_{i}} \omega H_{i}, \text { and }
$$

$$
\Omega_{H_{i}}^{*}=\left(\begin{array}{cc}
\Gamma_{H_{i}} & \Delta_{H_{i}}^{T} \\
\Delta_{H_{i}} & \bar{\Omega}_{H_{i}}
\end{array}\right)
$$

Proof. The proof follows from Theorem 2.6.

Theorem 4.1. (The lower convex order bound) The lower convex order bound $S_{l}$ which is used to approximate the distribution function of the sum $S=\sum_{i=0}^{n-1} \alpha_{i} e^{Z_{i}}$ is given by

$$
S_{l}=\sum_{i=0}^{n-1} \alpha_{i} \exp \left(\mu_{H_{i}}+\frac{1}{2} \Omega_{H_{i}}\right) \frac{\Phi_{m n}\left(\gamma_{H_{i}}+\Delta_{H_{i}}^{T} \omega_{H_{i}} ; \Gamma_{H_{i}}\right)}{\Phi_{m n}\left(\gamma_{H_{i}} ; \Gamma_{H_{i}}\right)}
$$

with $\mu_{H i}, \Omega_{H i},{ }_{H i}, \Delta_{H i}, \omega_{H i}$, and $\Gamma_{H i}$ defined in Lemma 4.5 .

Proof. By Lemma 3.1 the distribution of $\sum_{i=0}^{n-1} \alpha_{i} e^{Z_{i}}$ is approximated by the distribution of the sum $\sum_{i=0}^{n-1} \mathbb{E}\left[\alpha_{i} e^{Z_{i}} \mid \Lambda=\sum_{i=0}^{n-1} \nu_{i} Z_{i}\right]$

Thus the lower convex order bound $S_{l}$ is given by 


$$
\begin{aligned}
S_{l} & =\sum_{i=0}^{n-1} \mathrm{E}\left[\alpha_{i} \mathrm{e}^{Z_{i}} \mid \Lambda=\sum_{i=0}^{n-1} v_{i} Z_{i}\right] \\
& =\sum_{i=0}^{n-1} \alpha_{i} \mathrm{E}\left[\mathrm{e}^{Z_{i}} \mid \Lambda=\sum_{i=0}^{n-1} v_{i} Z\right]
\end{aligned}
$$

The expectation in (4.3) is the m.g.f of a random varible $y$ evaluated at $t=1$ where $y$ is distributed as

$$
S U N_{1, m n}\left(\boldsymbol{\mu}_{H_{i}}, \gamma_{H_{i}}, \bar{\omega}_{H_{i}}, \Omega_{H_{i}}^{*}\right)
$$

From Theorem 2.1, this m.g.f is obtained as

$$
\left.M_{y}(1)=\exp \mu_{H_{i}}+\frac{1}{2} \Omega_{H_{i}}\right) \frac{\left.\Phi_{n m} \gamma_{H_{i}}+\Delta_{H_{i}}^{T} \omega_{H_{i}} ; \Gamma_{H_{i}}\right)}{\Phi_{m n}\left(\gamma_{H_{i}} ; \Gamma_{H_{i}}\right)} .
$$

Therefore from (4.3) the convex lower order bound is

$$
\left.S=\sum \alpha_{i} \exp \mu_{H_{i}}+{ }_{2}^{1} \Omega_{H_{i}}\right) \begin{array}{ll}
\Phi_{m n} & \begin{array}{l}
\gamma_{H_{i}}+\Delta_{H_{i}}^{T} \omega_{H_{i}} ; \Gamma_{H_{i}} \\
\Phi
\end{array} \\
\Phi \quad(\gamma \quad ; \quad)
\end{array}
$$

\section{Lower bound in multi-asset case}

In the previous section we consider only one asset while deriving the distribution of terminal wealth. In the same fashion it is also possible to find the lower bound when the portfolio consists of multiple assets including risk-free and risky assets. Throughout this section we will assume that the portfolio has one riskfree asset (e.g cash account) and multiple risky assets (e.g stock funds). Following the previous section we will derive the lower bound step by step. However, we will have to redefine some variables to accommodate the case of multiple assets.

Let $Z_{j}^{i}$ be the sum of returns of one unit of capital invested at time $\mathrm{t}=\mathrm{j}$ to the final time $\mathrm{t}=\mathrm{n}$ of assest $\mathrm{i}, \mathrm{i}$ $=1, \ldots, \mathrm{q}$, that is

$$
\mathrm{Z}_{j}^{i}=\sum_{k=j+1}^{n} Y_{k}^{i}
$$

and the terminal wealth $S(\pi)$ is given by

$$
S(\pi)=\sum_{i=1}^{q} \sum_{j=0}^{n-1} \pi i \alpha j \exp (Z j i)+\sum_{j=0}^{n-1} \pi 0 \alpha j \exp ((n-j) r),
$$

where $\pi=\left(\pi_{1}, \ldots, \pi_{q}\right)^{T}$ is the vector of proportions of savings amounts in the risky assets and $\pi_{0}$ is the weight in the risk-free asset.

Lemma 5.1. (Joint distribution of $Y=\left(\boldsymbol{Y}_{1}^{T}, \ldots, \boldsymbol{Y}_{n}^{T}\right)^{T}$ ) Let the joint returns random vector $Y_{k}, k=1, \ldots, n$ be iid distributed as

$$
\operatorname{SUN}_{q, m}\left(\mu, \gamma, \omega^{-}, \Omega^{*}\right), \text { where } \Omega^{*}=\left(\begin{array}{cc}
\Gamma & \Delta^{T} \\
\Delta & \bar{\Omega}
\end{array}\right)
$$

Then the vector of log returns $Y=\left(\boldsymbol{Y}_{1}^{T}, \ldots, \boldsymbol{Y}_{n}^{T}\right)^{T}$ is distributed as 


$$
S U N_{n q, m n}\left(\boldsymbol{\mu}_{Y}, \gamma_{Y}, \overline{\boldsymbol{\omega}}_{Y}, \Omega_{Y}^{*}\right)
$$

where

$$
\mu_{Y}=\mathbf{1}_{n} \otimes \mu, \gamma_{Y}=\mathbf{1}_{n} \otimes \gamma, \omega_{Y}=I_{n} \otimes \omega, \omega_{Y}^{-}=\omega_{Y} \otimes \mathbf{1}_{n}
$$

and

$$
\Omega_{Y}=I_{n} \otimes \Omega, \quad \bar{\Omega}_{Y}=I_{n} \otimes \bar{\Omega}, \quad \Delta_{Y}=I_{n} \otimes \Delta, \quad \Gamma_{Y}=I_{n} \otimes \Gamma, \quad \Omega_{Y}^{*}=\left(\begin{array}{cc}
\Gamma_{Y} & \Delta_{Y}^{T} \\
\Delta_{Y} & \bar{\Omega}_{Y}
\end{array}\right)
$$

Proof. The proof follows from Corollary 2.2

Lemma 5.2. (Distribution of $Z=\left(\boldsymbol{Z}_{1}^{T}, \ldots, \boldsymbol{Z}_{q}^{T}\right)^{T}$ ) Let $Z=\left(\boldsymbol{Z}_{1}^{T}, \ldots, \boldsymbol{Z}_{q}^{T}\right)^{T}$ be the vector of accumulated returns, where $Z_{i}=\left(Z_{0}^{i}, \ldots, Z_{n-1}^{i}\right), i=0, \ldots, q$. Let $T_{j}^{i}$ be an $m$. $n$ dimensional row vector of $0^{0} s$ except in the $(i+q(j+k))$ th positions, $k=0,1, \ldots, n-(j+1)$ where they are $1^{0} s$ and let $T$ be a matrix whose rows are defined by vectors $T_{j}^{i}$. Then the distribution of $Z$ is

where

$$
S U N_{n q, m n}\left(\boldsymbol{\mu}_{Z}, \boldsymbol{\gamma}_{Z}, \overline{\boldsymbol{\omega}}_{Z}, \Omega_{Z}^{*}\right)
$$

$$
\mu_{Z}=T \boldsymbol{\mu}_{Y}, \quad \gamma_{Z}=\gamma_{Y}, \omega_{Z}=T \omega_{Y} T^{T}, \quad \overline{\boldsymbol{\omega}}_{Z}=\omega_{Z} \mathbf{1}_{n^{\prime}}
$$

and

$$
\Omega_{Z}=T \Omega_{Y} T^{T}, \quad \bar{\Omega}_{Z}=\omega_{Z}^{-1} \Omega_{Z} \omega_{Z}^{-1}, \quad \Delta_{Z}=\left(T \Omega_{Y} T^{T}\right)^{-1} T \omega_{Y} \Delta_{Y}, \quad \Gamma_{Z}=\Gamma_{Y}, \quad \Omega_{Z}^{*}=\left(\begin{array}{cc}
\Gamma_{Z} & \Delta_{Z}^{T} \\
\Delta_{Z} & \bar{\Omega}_{Z}
\end{array}\right) .
$$

Proof. The proof follows from Theorem 2.3 with T being the matrix of coefficients.

As in the single asset case, we use "Taylor-based" approach for choosing the random variable $\Lambda$. The random variable is accommodated to the multi asset case in the following way:

$$
\begin{aligned}
& q \quad n-1 \\
& \Lambda(\pi)=\mathrm{XX} v_{j i}(\pi) Z_{j i} \\
& i=0 j=0
\end{aligned}
$$

with the choice of the coefficents $v_{j}^{i}$, as $v_{j}^{i}(\pi)=\pi_{i} \alpha_{j} e^{\mathrm{E}[Z j i]}$. If the random variables $Y_{k}, k=1, \ldots, n$ are iid then the coefficients $v_{j}^{i}$ is given by $v_{j}^{i}(\pi)=\pi_{i} \alpha_{j} e^{\mathrm{E}[Z j i]}=\pi_{i} \alpha_{j} e^{(n-j) \mathrm{E}[Y i]}$, where $\mathrm{E}\left[Y^{i}\right]$ denotes the expectation of the $i$ th marginal distribution of the random vector $Y_{j}$.

Lemma 5.3. (Distribution of $\Lambda$ ) Let the random variable $\Lambda(\pi)$ be defined by

$$
\Lambda=\sum_{i=0}^{q} \sum_{j=0}^{n-1} \nu_{j}^{i}(\pi) Z_{j}^{i} \text { and let } V=\left(V_{1}, \ldots V_{q}\right) \text {, where } V_{i}=\left(\nu_{0}^{i}(\pi), \ldots \nu_{n-1}^{i}(\pi)\right), i=1, \ldots, q \text {. }
$$

Then the distribution of $\Lambda(\pi)$ is

$$
S U N_{1, m n}\left(\mu_{\Lambda}, \gamma_{\Lambda}, \bar{\omega}_{\Lambda}, \Omega_{\Lambda}^{*}\right)
$$

where

$$
\mu \Lambda=V \mu Z, \gamma \Lambda=\gamma Z, \quad \omega \Lambda=V \omega Z V t \omega^{-} Z=\omega \Lambda 1,
$$

and 


$$
\Omega_{\Lambda}=\boldsymbol{V} \Omega_{Z} \boldsymbol{V}^{T}, \quad \bar{\Omega}_{\Lambda}=\omega_{\Lambda}^{-1} \Omega_{\Lambda} \omega_{\Lambda}^{-1}, \quad \Delta_{\Lambda}=\left(\boldsymbol{V} \Omega_{Z} \boldsymbol{V}^{T}\right)^{-1} \boldsymbol{V} \omega_{Z} \Delta_{Z}, \quad \Gamma_{\Lambda}=\Gamma_{Z}, \quad \Omega_{\Lambda}^{*}=\left(\begin{array}{cc}
\Gamma_{\Lambda} & \Delta_{\Lambda}^{T} \\
\Delta_{\Lambda} & \bar{\Omega}_{\Lambda}
\end{array}\right)
$$

Proof. The proof follows from the Theorem 2.3 with $V$ being the matrix of coefficients.

Lemma 5.4. (Joint distribution of $\Lambda(\pi)$ and $Z_{j}^{i}$ ) Let $M_{j}^{i} \in<^{2 \times 9 \cdot n}$ be a matrix with the first row as $V$ and the second row of 0 's except in column $i \cdot n-(n-j-1), i=1, \ldots, q, j=0, \ldots, n-1$ where the 0 is replaced by 1 . Then the distribution of $\left.X_{j}^{i}=\Lambda(\pi), Z_{j}^{i}\right)^{T}$ is

$$
S U N_{2, m n}\left(\boldsymbol{\mu}_{X_{j}^{i}}, \gamma_{X_{j}^{i}}, \overline{\boldsymbol{\omega}}_{X_{j}^{i}}, \Omega_{X_{j}^{i}}^{*}\right)
$$

where

$$
\begin{gathered}
\mu X_{j i}=M_{j i} \mu z, \gamma X_{j i}=\gamma Z, \quad \omega X_{j i}=M_{j i} \omega Z M_{j T i} \omega^{-} X_{j i}=\omega X_{j i 1} 1_{2}, \\
\Omega_{X_{j}^{i}}=M_{j}^{i} \Omega_{Z} M_{j^{i}}^{T}, \quad \bar{\Omega}_{X_{i}}=\omega_{X_{j}^{i}}^{-1} \Omega_{X_{j}^{i}} \omega_{X_{j}^{i}}^{-1}, \quad \Delta_{X_{j}^{i}}=\left(M_{j}^{i} \omega_{X_{j}^{i}} M_{j^{i}}^{T}\right)^{-1} M_{j}^{i} \omega_{X_{j}^{i}} \Delta_{Z}, \quad \Gamma_{X_{j}^{i}}=\Gamma_{Z}
\end{gathered}
$$

and

$$
\Omega_{X_{j}^{i}}^{*}=\left(\begin{array}{cc}
\Gamma_{X_{j}^{i}} & \Delta_{X_{j}^{i}}^{T} \\
\Delta_{X_{j}^{i}} & \bar{\Omega}_{X_{j}^{i}}
\end{array}\right)
$$

Proof. The proof follows from Theorem 2.3 with $M_{j}{ }^{i}$ being the matrix of coefficients.

Lemma 5.5. (Conditional distribution of $Z_{j}^{i}$ given $\Lambda(\pi)$ ) Let $\mu_{x} j i, \mathbf{\Omega}_{x j}^{-} i, \omega_{x j}^{-} i$ and $\Delta_{x} j i$ be partitioned as in theorem 2.5. Then the conditional distribution of $H_{j}{ }^{i}=\left(Z_{j}^{i} \mid \Lambda=\lambda\right)$ is given by

$$
S U N_{1, m n}\left(\mu_{H_{j}^{i}}, \gamma_{H_{j}^{i}}, \bar{\omega}_{H_{j}^{i}}, \Omega_{H_{j}^{i}}^{*}\right)
$$

where

$$
\mu H_{j i}=\mu 2+\Omega_{21} \Omega-211(\lambda-\mu 1), \gamma_{j i i}=\gamma_{j i}+\Delta 1 T \Omega^{-}-111 \omega_{2-1}(\lambda-\mu 1), \omega^{-}{ }_{j i}=\omega^{-} 1,
$$

$\Gamma_{H j i}=\Gamma X_{j i}-\Delta T 1 \Omega^{-}{ }_{-111} \Delta 1, \Delta H_{j i}=\Delta 2-\Omega 21^{-} \Omega^{-}{ }_{-111} \Delta 1, \Omega^{-}{ }_{H j i}=\Omega^{-}{ }_{22}-\Omega^{-}{ }_{21} \Omega^{-}{ }_{-111} \Omega^{-}{ }_{12}, \Omega_{H_{j i}}=\omega_{H_{j i}} \Omega^{-}{ }_{H j i} \omega H_{j j}$, and

$$
\Omega_{H_{j}^{i}}^{*}=\left(\begin{array}{cc}
\Gamma_{H_{j}^{i}} & \Delta_{H_{j}^{i}}^{T} \\
\Delta_{H_{j}^{i}} & \bar{\Omega}_{H_{j}^{i}}
\end{array}\right)
$$

Theorem 5.1. (The lower convex order bound) The lower convex order bound $S_{l}(\pi)$ to approximate the distribution function of the sum $S(\boldsymbol{\pi})=\sum_{i=1}^{q} \sum_{j=0}^{n-1} \pi_{i} \alpha_{j} \exp \left(Z_{j}^{i}\right)+\sum_{j=0}^{n-1} \pi_{0} \alpha_{j} \exp ((n-j) r) i s$ given by

$$
\left.S_{l}(\pi)=\sum_{i=1}^{q} \sum_{j=0}^{n-1} \pi_{i} \alpha_{j} \exp \mu_{H_{j}^{i}}+\frac{1}{2} \Omega_{H_{j}^{i}}\right) \frac{\left.\Phi_{m n} \gamma_{H_{j}^{i}}+\Delta_{H_{j}^{i}}^{T} \omega_{H_{j}^{i}} ; \Gamma_{H_{j}^{i}}\right)}{\Phi_{m n}\left(\gamma_{H_{j}^{i}} ; \Gamma_{H_{j}^{i}}\right)}+\sum_{j=0}^{n-1} \pi_{0} \alpha_{j} \exp ((n-j) r)
$$

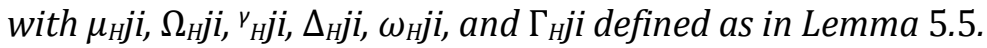

Proof. The proof is same as the one given in Theorem 4.1 . 


\section{Numerical results}

In this section we numerically illustrate the accuracy of the approximations obtained in the previous two sections by using two examples. We do not use a real data set to analyze the approximations since making inference on the unified skew normal distribution is difficult due to its large number of parameters, instead we use a hypothetical data to evaluate the accuracy. For the estimation of parameters of SUN we refer to Gupta and Aziz (2012).

In the first example, the final wealth of yearly savings distributed as log unified skew normal random variables is computed using Monte Carlo method and the method presented in section 4.

For $n=20$, at every period $i, i=0, \ldots, n-1$, consider the yearly savings amounts $\alpha i=1, i=0, \ldots, 19$. That is, at the beginning of each year one unit of savings amount is invested in the considered asset. At time $\mathrm{i}=\mathrm{n}$ the invested amount $\alpha \mathrm{n}=0$, i.e, no contribution is made at the final period. The returns are considered to be independently and indentically distributed SUN random variables with parameters $\mathrm{m}=\mathrm{d}=1, \mu=0.02, \gamma=0$, $\omega=1, \Omega=\Omega^{-}=0.03, \Gamma=1$, and $\Delta=0.97$. The results for some selected quantiles of the distribution function of the terminal wealth obtained by the Monte Carlo simulation (denoted by MCB) and from the convex lower bound (CLB) are presented in Table 1.

Table 1: Comparison of the selected quantiles of the distribution of the final wealth in single asset case

\begin{tabular}{lclc}
\hline \hline$p$ & $M C B$ & \multicolumn{2}{l}{ CLB Relative de viation } \\
\hline 0.01 & 22.5789 & 22.8575 & $1.23 \%$ \\
0.025 & 25.9758 & 26.1992 & $0.86 \%$ \\
0.05 & 29.7387 & 29.9730 & $0.78 \%$ \\
0.95 & 152.9541 & 151.5890 & $-0.89 \%$ \\
0.975 & 184.0342 & 182.4662 & $-0.85 \%$ \\
0.99 & 226.9628 & 222.1930 & $-2.10 \%$ \\
\hline
\end{tabular}

The simulated results are obtained from 5000 random paths. The relative deviations of the approximated values from the Monte Carlo simulation are computed as follows:

$$
\frac{Q_{p}\left[S_{n}^{l}\right]-Q_{p}\left[S_{n}^{M C}\right]}{Q_{p}\left[S_{n}^{M C}\right]} \times 100
$$

Comparing the results obtained with the Monte Carlo simulations, all the lower bound approximations seem to perform reasonably well, in fact some of them are excellent. The approximations lose some precision in the tails of the distribution.

In the second example, we illustrate the approximations for a portfolio consisting of two risky assets and one risk free asset. We consider the same savings amount as in the first example (that is, $\alpha \mathrm{i}=1, \mathrm{i}=1, \ldots, \mathrm{n}$ ) and the weights are assigned as follows: $19 \%$ in the risk-free asset, $45 \%$ in the first risky asset and the remaining $36 \%$ will be invested in the second risky asset. In addition, the yearly return of the risk-free asset is considered 
to be 0.03 . The parameters of the joint distribution of the risky assets are chosen to be $\mathrm{m}=1, \mathrm{~d}=2, \mu=$ $(0.06,0.1) \mathrm{T}, \gamma=0, \omega=\mathrm{I} 2, \Omega=\Omega^{-}=\left(\begin{array}{ll}0.01 & 0.01 \\ 0.01 & 0.04\end{array}\right), \Gamma=1$, and $\Delta=(-0.95,-0.97)^{T}$.

The results for the distribution function of the terminal wealth obtained by the Monte Carlo simulation and from the convex lower bound are presented in Table 2. As for the single asset case, the simulated results are obtained from 5000 random paths.

From Table 2, it is noted that the approximation is still reasonably good when we consider a portfolio. The approximations at the tails of the distribution lose more precisions compared to the single asset situation. One of the reasons might be that in the multi-asset case we include an extra risky asset thus making the number of log unified skew normal random variables double compared to the single asset case. However, the approximations will surely improve with a better choice of the conditioning random variable $\Lambda$.

Table 2: Comparison of the selected quantiles of the distribution of the final wealth in multi asset case

\begin{tabular}{lcll}
\hline \hline$p$ & $M C B$ & \multicolumn{2}{l}{$C L B$ Relative de viation } \\
\hline 0.01 & 19.4143 & 19.7961 & $1.97 \%$ \\
0.025 & 22.0037 & 22.3248 & $1.46 \%$ \\
0.05 & 24.5388 & 24.7480 & $0.85 \%$ \\
0.95 & 124.8726 & 124.3719 & $-0.40 \%$ \\
0.975 & 152.1901 & 150.5944 & $-1.05 \%$ \\
0.99 & 196.4655 & 191.0344 & $-2.76 \%$ \\
\hline
\end{tabular}

\section{Conclusion}

Extending the works of Marin-Solano et al. (2010), and Roch and Valdez (2011), we provide analytical expressions and numerical illustrations to approximate the distribution function of terminal wealth. From the numerical illustrations, we find that the VaR describing the terminal wealth obtained from lower convex order bound are competitive to those obtained from a more time consuming Monte Carlo method. The risk measure could be improved by other suitable choice of conditioning random variable $\Lambda$ and considering other risk measures such as conditional tail expectation.

\section{Acknowledgement}

This work was supported in part by an allocation of computing time from the Ohio Supercomputer Center. 


\section{References}

[1] Arellano-Valle, R. B. and Azzalini, A. (2006). On the unification of families of skew-normal distributions. Scand. J. Statist., 33, 561-574.

[2] Azzalini, A. and Dalla Valle, A. (1996). The multivariate skew-normal distribution. Biometrika, 83, 715-726.

[3] Dhaene, J., Denuit, M., Gooverts, M., Kass, R., and Vyncke, D. (2002a). The Concept of Comonotonicity in Actuarial Science and Finance: Theory. Insurance: Mathematics and Economics, 31, 3-33.

[4] Dhaene, J., Denuit, M., Gooverts, M., Kass, R., and Vyncke, D. (2002b). The Concept of Comonotonicity in Actuarial Science and Finance: Applications. Insurance: Mathematics and Economics, 31, 133-161.

[5] Domingnez-Molina, J. A., Gonzalez-Farias, G., and and Gupta, A. K. (2001). General multivariate skew normal distribution. Department of Mathematics and Statistics, Bowling Green State University, Tecnical Report No.01-09.

[6] Gupta, A. K., Gonzalez-Farias, G., and Dominguez-Molina, J. A. (2004). A multivariate skew normal distribution. J. Multivariate Anal. 89, 181-190.

[7] Gupta, A. K. and Aziz, M. A. (2012). Estimation of parameters of the unified skew normal distribution using method of weighted moments. Journal of Statistical Theory and Practice, 6 (3), 402-416.

[8] Gupta, A. K., Aziz, M. A and Ning, W. (2013). On some properties of unified skew normal distribution. Journal of Statistical Theory and Practice.7(3), 480-495.

[9] Gupta, A. K., Nagar, D. K. (2000). Matrix Variate Distributions. Chapman and Hall/CRC, New York.

[10] Horn, R. A., Johnson, C. R. (1991). Topics in Matrix Analysis. Cambridge University Press, Cambridge.

[11] Kaas, R., Goovaerts , M.J., Dhaene, J. and Denuit, M. (2001). Modern Actuarial Risk Theory. Kluwer Academic Publishers, Dordrecht.

[12] Marin-Solano, J., O. Roch, J. Dhaene, C. Ribas, M. Bosch-Princep, and S. Vanduffel (2010). Buy and hold strategies and comonotonic approximations. Belgian Actuarial Bulletin 9(1), 17-28.

[13] Roch, O. and Valdez, E. (2011). Lower convex order bound approximations for sums of logskew normal random variables. Applied Stochastic Models in Business and Industry. 27(5) , 487-502

[14] Vanduffel, S., Chen, X.; Dhaene, J.; Goovaerts, M.; Henrard, L.; and Kass, R. (2008). Optimal approximations for risk measures of sums of lognormals based on conditional expectations. Journal of Computational and Applied Mathematics, 221(1), 202-218. 